(2) Open Access Full Text Article

\title{
Inappropriateness of medication prescriptions about chronic kidney disease patients without dialysis therapy in a Chinese tertiary teaching hospital
}

\author{
This article was published in the following Dove Press journal: \\ Therapeutics and Clinical Risk Management \\ 12 October 2016 \\ Number of times this article has been viewed
}

\author{
Ping Yang \\ $\mathrm{Na}$ Chen \\ Rong-Rong Wang \\ Lu Li \\ Sai-Ping Jiang \\ Department of Pharmacy, The \\ First Affiliated Hospital, College \\ of Medicine, Zhejiang University, \\ Hangzhou, People's Republic of China
}

Correspondence: Sai-Ping Jiang Department of Pharmacy, The First Affiliated Hospital, College of Medicine, Zhejiang University, 79 Qingchun Road, Hangzhou, 3I0003, People's Republic of China

Tel +86 57| $872334 \mid I$

Fax +86 57I 8723 34II

Email j5 I45@I26.com
Background: With the increasing incidence rate of chronic kidney disease (CKD), inappropriate use of medicine in CKD patients is an important issue, as it may cause adverse effects in patients and progression to chronic renal failure.

Objective: The aim of this study is to assess the frequency of inappropriate medicine use among CKD patients.

Methods: A cross-sectional study was conducted from November 1 to December 1, 2014 in a Chinese teaching tertiary hospital. All medication prescriptions for CKD patients with serum creatinine level above normal value were enrolled. The prescriptions, including unreasonable dosage, contraindicated, and cautiously used medicines in CKD patients, were evaluated and the related medications were also analyzed and classified.

Results: Two hundred and two patients were included, and a total of 1,733 lines of medication prescriptions were evaluated. The prevalence of inappropriate medication prescriptions in CKD patients was $15.18 \%$, of which, unreasonable dosage $(n=56)$, contraindicated $(n=46)$, and cautiously used medicines $(n=161)$ accounted for $3.23 \%, 2.65 \%$, and $9.29 \%$, respectively. Spearman's rank correlation coefficient implied that there was a significant correlation between the severity of renal insufficiency and frequency of inappropriate medication prescriptions $(P=0.02, r=0.056)$. Among the inappropriate medication prescriptions, nutraceutical and electrolytes $(n=65,24.71 \%)$, cardiovascular drugs $(n=61,23.19 \%)$, and antimicrobial drugs $(n=55$, 20.91\%) represented the top three medicine categories in CKD patients.

Conclusion: The study confirmed that inappropriate medication prescriptions were prevalent in CKD patients. Improving the quality of medication prescriptions in CKD patients is necessary.

Keywords: inappropriateness of medication prescriptions, chronic kidney disease, unreasonable dosage medicines, contraindicated used medicines, cautiously used medicines

\section{Introduction}

Chronic kidney disease (CKD) is defined as abnormalities in kidney structure or function that have been present for over 3 months, with implications for health. ${ }^{1,2}$ All stages of CKD are associated with increased health care use and Medicare expenditure. ${ }^{3}$ At present, the incidence of CKD is rapidly increasing. ${ }^{4}$ It has been reported that there is one medication-related problem for every 2.7 dialysis patients. ${ }^{5}$ Despite widespread evidence in the literature, dosing errors in patients with CKD still occur at an alarming rate. ${ }^{6}$ 
Impaired renal function can have pronounced effects on the pharmacokinetics of many medicines as a result of alterations in absorption, distribution, metabolism, and excretion. The most common alteration is a decrease in drug excretion, resulting in increased plasma concentrations and heightened risk of drug toxicity. Therefore, there is an increased risk of drug-related problems such as the use of contraindicated medicines and inappropriate dosages, with potentially adverse outcomes. ${ }^{7,8}$ It is essential to select the appropriate medicine and individualize the dosage in order to avoid the occurrence of adverse drug reaction (ADR).

For CKD patients, inappropriate medication may be generated when the medicine has not been adjusted to appropriate dosage or some nephrotoxic medicine has been prescribed. As a result, the risk of ADR further increases. ${ }^{9}$ Compared with patients with normal renal function, CKD patients are more prone to experiencing ADRs. ${ }^{10,11}$ Appropriate medicine selection and dosage for patients with CKD are important to avoid unwanted medicine effects and ensure optimal patient outcomes. Efforts to reduce dosing errors can lower the rate of ADR, reduce costs, and improve the overall delivery of health care. ${ }^{12-15}$

Up to now, a number of studies have been reported on the use of medicines in patients with renal dysfunction. However, they only focused on a certain type of medicine. Investigations of antibiotics were most reported for renal insufficiency, ${ }^{16-21}$ followed by nonsteroidal antiinflammatory drugs and angiotensin-converting enzyme inhibitors. $^{22,23}$ As reported in China, dosage errors for antibiotics prescribed for CKD patients were in the range of $38.85 \%-60.3 \%{ }^{20,21}$ To the best of the authors' knowledge, there was no study that comprehensively analyzed the inappropriate medicine use in CKD patients at tertiary teaching hospitals. Therefore, the authors retrospectively investigated medicine prescriptions in the hospital's CKD patients, and then the inappropriate medication prescriptions were analyzed and classified.

\section{Methods}

\section{Study design and patient selection}

A cross-sectional study was conducted in a Chinese tertiary teaching hospital from November 1 to December 1, 2014. The hospital is a comprehensive and information-based hospital with 2,500 beds, where a multidisciplinary team of diverse professionals provide a range of health-care services for approximately 114,000 inpatients each year. During the data collection period, all the patients hospitalized were included in this study. Data regarding prescribing of medications were collected from the hospital information system, then for the further data analysis.

Patients were included if they were: 1) CKD patients with serum creatinine level above the normal value according to the hospital's criterion of $45-84 \mu \mathrm{mol} / \mathrm{L}$ for women and 59-104 $\mu \mathrm{mol} / \mathrm{L}$ for men; 2) aged $\geq 18$ years; 3 ) had body mass index $\geq 18.5 \mathrm{~kg} / \mathrm{m}^{2}$ and $\leq 24.9 \mathrm{~kg} / \mathrm{m}^{2}$. The exclusion criteria were: patients receiving hemodialysis, peritoneal dialysis, or renal replacement therapy.

Ethical approval was acquired from the First Affiliated Hospital of ZheJiang University's research review committee. All patients enrolled in this study provided written informed consent for their information to be used for this study.

\section{Data collection and evaluation on appropriateness of prescriptions}

Data on the characteristics of patients and medication prescriptions were extracted from the hospital information system. Two clinical pharmacists independently collected data by using a structured format; any dispute was resolved through discussion or with the assistance of another clinical pharmacist. The content included demographic variables, name of the medication, dosage forms, dosages, frequency, and number of medicines prescribed. Demographic information about a patient, including gender, age, weight, and serum creatinine, was used to determine the creatinine clearance rate $(\mathrm{Ccr})$, which was calculated by using the Cockcroft-Gault equation:

$$
\begin{gathered}
\text { If male: } \mathrm{Ccr}=\frac{(140-\text { age }) \times \text { Weight }}{72 \times \mathrm{SCr}} \\
\text { If female: } \mathrm{Ccr}=\frac{(140-\text { age }) \times \text { Weight }}{72 \times \mathrm{SCr}} \times 0.85
\end{gathered}
$$

where Ccr is expressed in milliliters per minute, age in years, weight in kilograms, and serum creatinine in milligrams per deciliter. Despite the limitation of this equation, it is the most prevalent method for determining medicine dosage based on kidney function in medication instruction (MI). Then stages of CKD were graded according to Ccr by the National Kidney Foundation Kidney Disease Outcomes Quality Initiative guidelines.

To evaluate the appropriateness of prescriptions for each enrolled patient, the prescribed medicines were compared with MI approved by the China Food and Drug Administration. Evaluation of the inappropriateness of prescriptions was based on three aspects, namely unreasonable medicine dosage, contraindicated, and cautiously used medicine. Unreasonable medicine dosages imply medicines 
prescribed at a dose that is more or less than what is recommended by MI. Contraindicated used medicines imply the medicines should have been avoided in CKD patients, while cautiously used medicines imply the medicines should be used carefully in the CKD patients. Thereafter, the prevalence of inappropriate prescriptions in CKD patient and the related medications were classified and analyzed.

\section{Statistical analyses}

Descriptive statistics were performed, response associations between stage of CKD and frequency of inappropriate medication prescriptions were determined by using Spearman's rank correlation coefficient $(r)$, with $P$-value $<0.05$ as significant correlation. The analysis was performed by Stata 12.0 software (StataCorp LP, College Station, TX, USA).

\section{Results}

\section{Patient characteristics}

In all, 9,589 hospitalized patients were included in the study by primary screening. Then, 202 CKD patients involving 1,733 lines of medicine prescriptions were enrolled on the basis of inclusion and exclusion criteria in this study. Basic characteristics of included patients are presented in Table 1. The majority of the $202 \mathrm{CKD}$ patients $(64.36 \%)$ were male, with a mean age of $64.83 \pm 15.46$ years, and the average number of medications per patient was $8.58 \pm 5.06$. According to the classification of CKD, $54.46 \%$ CKD patients were in the G3 stage of renal dysfunction. Moreover, the ward distribution of 202 enrolled patients was investigated. As shown in Figure 1, 23.76\% patients were treated in the nephrology department.

\section{Frequency of inappropriate prescriptions in CKD patients}

As exhibited in Table 2, among the 1,733 lines of medicine prescriptions for 202 CKD patients, 263 lines (15.18\%) were inappropriate prescriptions. Among these inappropriate prescriptions, unreasonable medicine dosages, contraindicated, and cautiously used medicines accounted for 56 (3.23\%), 46 (2.65\%), and 161 (9.29\%), respectively. This study found that the inappropriateness of unreasonable medicine dosages and contraindicated used medicines were mostly administered in the G4 and G5 stages of CKD, while the cautiously used medicines were administered in all the stages of CKD.

The frequency of inappropriate medication prescriptions in patients with various severity of CKD was also analyzed, as shown in Table 2. Spearman's rank correlation coefficient implied that there were significant correlations between the severity of renal insufficiency and frequency of inappropriate medication prescriptions $(P=0.02, r=0.056)$.

\section{Medications related with inappropriate prescriptions in CKD patients}

Among the inappropriate medication prescriptions in CKD patients, nutraceutical and electrolytes ( $\mathrm{n}=65,24.71 \%)$, cardiovascular drugs $(\mathrm{n}=61,23.19 \%)$, and antimicrobial drugs $(n=55,20.91 \%)$ represented the three top medicine categories. As shown in Figure 2, antimicrobial drugs ( $\mathrm{n}=50$, $89.29 \%$ ) were the most common category of medicines associated with unreasonable dosage, followed by cardiovascular drugs $(n=4,7.14 \%)$. Endocrine and metabolic drugs $(n=17,36.96 \%)$ were the most common contraindicated drug categories used in renal dysfunction; next were central nervous system $(n=6,13.04 \%)$ and hematologic $(n=6$, $13.04 \%)$ drug categories. Nutraceuticals and electrolytes $(\mathrm{n}=60,37.27 \%)$, diuretics $(\mathrm{n}=28,17.39 \%)$, and cardiovascular drugs ( $n=24,14.91 \%)$ were the top three categories of medicines that correlated with cautiously used medicines in CKD patients.

Taking specific medicines into consideration, carbohydrate-electrolyte solution ( $\mathrm{n}=17,6.46 \%)$, potassium chloride $(n=16,6.08 \%)$, and spironolactone $(n=14,5.32 \%)$ were the top three medicines of inappropriate medication

Table I Characteristics of enrolled CKD patients

\begin{tabular}{lllll}
\hline $\begin{array}{l}\text { Stage of CKD } \\
(\mathbf{n}, \%)\end{array}$ & Gender, $\mathbf{n}(\%)$ & Age, & & $\begin{array}{l}\text { Medicines used, } \\
\text { mean } \pm \text { SD }\end{array}$ \\
\cline { 2 - 3 } mean \pm SD & Male & $9(31.03)$ & $64.56 \pm 15.46$ & $8.46 \pm 5.00$ \\
G2 (29, 14.36) & $20(68.97)$ & $20(30.77)$ & $64.54 \pm 15.39$ & $8.49 \pm 5.02$ \\
G3a $(65,32.18)$ & $45(69.23)$ & $15(33.33)$ & $64.80 \pm 15.45$ & $8.54 \pm 5.07$ \\
G3b $(45,22.28)$ & $30(66.67)$ & $17(38.64)$ & $64.76 \pm 15.47$ & $8.45 \pm 4.99$ \\
G4 (44, 21.78) & $27(61.36)$ & $11(57.89)$ & $64.12 \pm 15.70$ & $8.66 \pm 5.12$ \\
G5 $(19,9.40)$ & $8(42.11)$ & $72(35.64)$ & $64.83 \pm 15.46$ & $8.58 \pm 5.06$ \\
Total $(202,100)$ & $130(64.36)$ & & & \\
\hline
\end{tabular}

Abbreviations: CKD, chronic kidney disease; SD, standard deviation. 

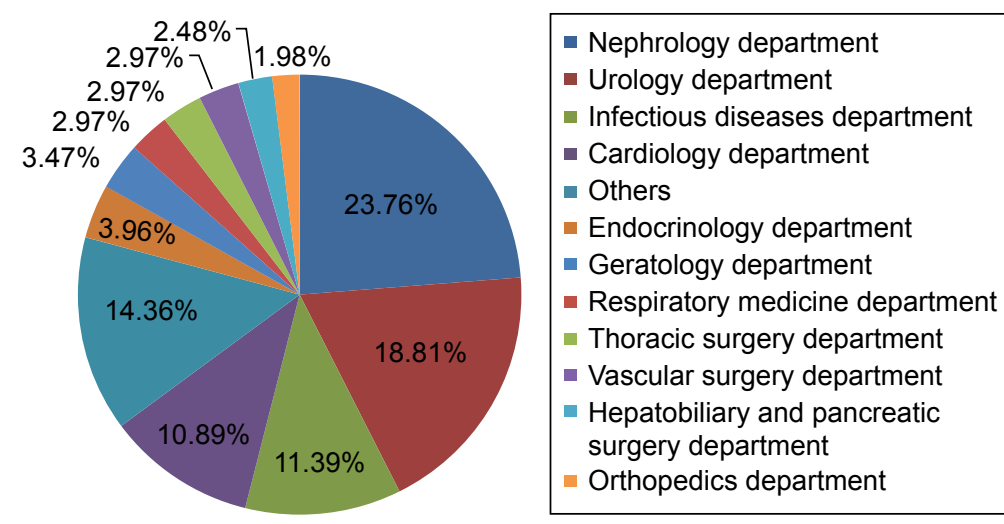

Figure I Ward distribution of enrolled CKD patients.

Abbreviation: CKD, chronic kidney disease.

prescriptions. As shown in Table 3, piperacillin-tazobactam $(\mathrm{n}=12,21.43 \%)$, acarbose $(\mathrm{n}=7,15.22 \%)$, and carbohydrateelectrolyte solution $(n=17,7.98 \%)$ were found to be the most common medications associated with inappropriate prescriptions in unreasonable dosage used, contraindicated, and cautiously used medicines, respectively. The evaluation on appropriateness of carbohydrate-electrolyte solution and potassium chloride was based on the serum electrolyte levels according to the standard in the hospital.

\section{Discussion}

Because most medicines are predominantly eliminated in urine, renal function is expected to play an important role in their disposition. Understanding and quantification of the impact of various degrees of kidney function on medicine exposure is important to help with appropriate medicine selection and dose adjustment decisions in CKD patients. However, reports on the analysis of inappropriate medication prescriptions in CKD patients remain limited. As far as the authors know, this study is the first to assess the frequency of inappropriate medication prescriptions in CKD patients in a Chinese tertiary teaching hospital.
Optimizing medicines dosage and choice in CKD could reduce the incidence of adverse drug events (ADEs)..$^{24,25}$ Hassan et al reported that hospital pharmacists can contribute to a reduction in the incidence of ADEs in patients with renal impairment. ${ }^{26} \mathrm{It}$ is possible that physicians pay more attention to patients' diagnosis than details of medication prescribing. However, impaired renal function can be associated with alterations of drug pharmacokinetics; awareness of proper medicine selection and dosage individualization is of critical importance, as it can avoid the occurrence of ADEs and the risk of therapeutic failure.

This study sheds light on a problem that cannot be ignored in CKD patients. According to this study, unreasonable medicine dosage $(n=56,3.23 \%)$, contraindicated $(n=46,2.65 \%)$, and cautiously used medicines ( $\mathrm{n}=161,9.29 \%$ ) were common inappropriate phenomenon in CKD patients. Spearman's rank correlation analysis revealed that the frequency of inappropriate medication prescriptions was positively corrected with the severity of renal insufficiency $(P=0.02, r=0.056)$. A possible explanation for this could be that the nature and opportunistic diagnosis of CKD resulted in the higher prevalence of inappropriate prescribing. ${ }^{27,28}$ Other contributing factors reported

Table 2 Inappropriate prescriptions in various severity CKD patients

\begin{tabular}{|c|c|c|c|c|c|}
\hline $\begin{array}{l}\text { Stage of CKD } \\
(n, \%)\end{array}$ & $\begin{array}{l}\text { Unreasonable } \\
\text { dosage, } n(\%)\end{array}$ & $\begin{array}{l}\text { Contraindicated } \\
\text { used medicines } \mathbf{n}(\%)\end{array}$ & $\begin{array}{l}\text { Cautiously used } \\
\text { medicines, } \mathbf{n}(\%)\end{array}$ & $\begin{array}{l}\text { Inappropriate medication } \\
\text { prescription, } \mathbf{n}(\%)\end{array}$ & $\begin{array}{l}\text { Total medication } \\
\text { prescription }(\mathrm{n})\end{array}$ \\
\hline G2 $(29,14.36)$ & I (0.57) & I $(0.57)$ & $19(10.86)$ & $21(12)$ & 175 \\
\hline G3a $(65,32.18)$ & $16(3.14)$ & $7(1.37)$ & $48(9.4 I)$ & 71 (13.92) & 510 \\
\hline G3b $(45,22.28)$ & II (2.79) & $5(1.27)$ & 31 (7.87) & 47 (II.93) & 394 \\
\hline G4 (44, 21.78$)$ & $25(5.68)$ & $21(4.77)$ & 48 (10.9I) & $94(21.36)$ & 440 \\
\hline G5 $(19,9.40)$ & $3(1.40)$ & $12(5.61)$ & $15(7.0 \mathrm{I})$ & $30(14.02)$ & 214 \\
\hline Total & $56(3.23)$ & $46(2.65)$ & $|6|(9.29)$ & $263(15.18)$ & I,733 \\
\hline
\end{tabular}

Note: Spearman's rank correlation analysis revealed that the frequency of inappropriate medication prescriptions was positively corrected with the severity of renal insufficiency $(P=0.02, r=0.056)$.

Abbreviation: CKD, chronic kidney disease. 


\section{A Percentage of prescriptions with unreasonable dosage medicines}

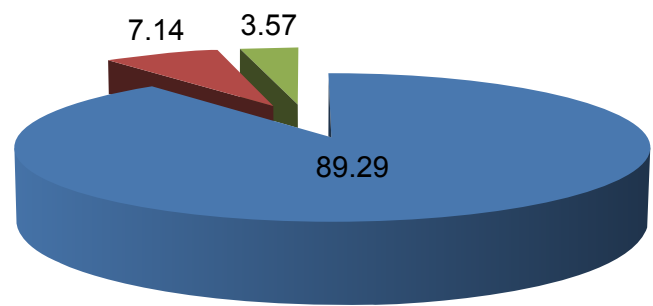

Anti-infective

- Cardiovascular

- Others

\section{B Percentage of prescriptions with contraindicated} used medicines
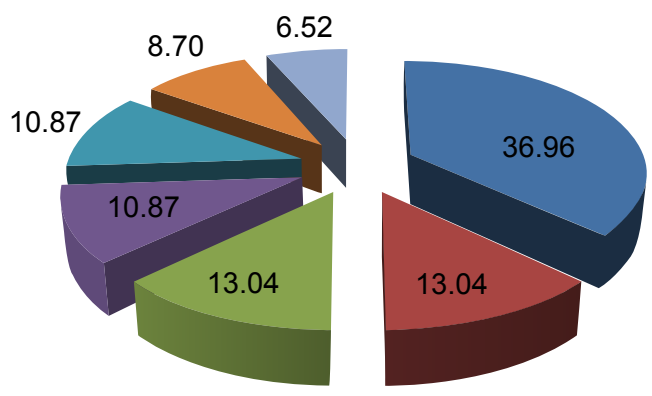

Endocrine and metabolic

- Hematologic

- Central nervous

- Cardiovascular

- Nutraceutical and electrolytes

- Anti-infective

- NSAIDs

\section{Percentage of prescriptions with cautiously used medicines}

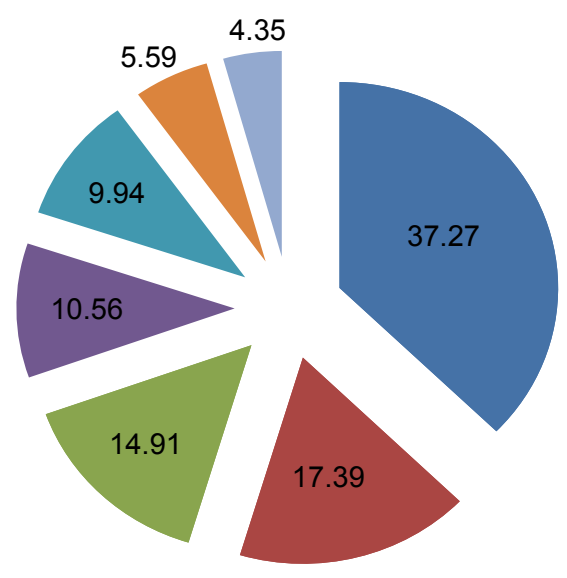

- Nutraceutical and electrolytes

- Diuretic

- Cardiovascular

- Others

- Endocrine and metabolic

- NSAIDs

- Digestive

Figure 2 Therapeutic category of medications with inappropriate prescription in CKD patients.

Notes: (A) Unreasonable dosage medicines; (B) contraindicated used medicines; (C) cautiously used medicines.

Abbreviations: CKD, chronic kidney disease; NSAIDs, nonsteroidal anti-inflammatory drugs.

Table 3 Top five medicines associated with inappropriate medication prescriptions in CKD patients

\begin{tabular}{|c|c|c|c|}
\hline \multirow[t]{2}{*}{ Ranking } & \multicolumn{3}{|l|}{ Frequency $(n, \%)$} \\
\hline & $\begin{array}{l}\text { Unreasonable dosage } \\
\text { medicines }(n=56)\end{array}$ & $\begin{array}{l}\text { Contraindicated used } \\
\text { medicines }(n=46)\end{array}$ & $\begin{array}{l}\text { Cautiously used medicines } \\
(n=2 \mid 3)\end{array}$ \\
\hline First & $\begin{array}{l}\text { Piperacillin-tazobactam } \\
(12,21.43 \%)\end{array}$ & $\begin{array}{l}\text { Acarbose } \\
(7,15.22 \%)\end{array}$ & $\begin{array}{l}\text { Carbohydrate-electrolyte solution } \\
(17,7.98 \%)\end{array}$ \\
\hline Second & Levofloxacin (7, 12.5\%) & Metformin $(3,6.52 \%)$ & Potassium chloride $(16,7.51 \%)$ \\
\hline Third & Ceftazidime $(6,10.71 \%)$ & Lamivudine $(3,6.52 \%)$ & Spironolactone $(14,6.57 \%)$ \\
\hline Fourth & Adefovir dipivoxil $(3,5.36 \%)$ & Colchicines $(3,6.52 \%)$ & Calcium gluconate $(10,4.69 \%)$ \\
\hline Fifth & Entecavir $(3,5.36 \%)$ & Rosuvastatin Calcium $(2,4.35 \%)$ & Isosorbide mononitrate $(8,3.76 \%)$ \\
\hline
\end{tabular}

Abbreviation: CKD, chronic kidney disease. 
included prescribers' poor knowledge of medications requiring dosage adjustment, underestimation of potential adverse events, and lack of evidence-based data to guide prescribers on dosage adjustments as well as lack of quantitative data in the available MI. ${ }^{29,30}$ It is necessary that physicians should consider patients' renal function when prescribing for CKD patients.

It was found that for unreasonable medicine dosage use, antimicrobial drugs accounted for the highest proportion. Most of the antimicrobial drugs, such as $\beta$-lactams, are eliminated by the kidneys in an unchanged condition, so dosage adjustment is frequently required in patients with renal insufficiency. However, antibiotic dosages in six prescriptions were lower than the recommendation of $\mathrm{MI}$, for example, piperacillin-tazobactam $(n=4)$ and cefuroxime $(n=1)$, these $\beta$-lactams antibiotics required dosage adjustment only when the Ccr level was $<40 \mathrm{~mL} / \mathrm{min}$ and $20 \mathrm{~mL} / \mathrm{min}$, respectively. In addition, metronidazole $(n=1)$, which is mainly metabolized in the liver and eliminated by the kidneys, needed no dosage adjustment under renal insufficiency. An incorrect dosage adjustment may result in lower dosage and poor treatment effect. One explanation that maybe advanced for this incorrect adjustment is that, the physician was not familiar with the pharmacokinetics of the prescribed medicines mentioned earlier. Correspondingly, the dosages of the other prescriptions $(n=44)$ were higher than the recommendation of MI, such as quinolones, cephalosporins, entecavir, adefovir dipivoxil, and so on, which are predominantly excreted by the kidneys; normal dosage may cause potential drug accumulation and adverse reactions.

It is worth noting that antidiabetic drugs were the most common contraindicated used medicines in CKD patients, such as acarbose and metformin. In CKD patients, metformin may cause life-threatening lactic acidosis, ${ }^{31}$ so current medicine labels regard the use of metformin as contraindicated in patients with serum creatinine level $>1.5 \mathrm{mg} / \mathrm{dL}$ for males and $1.4 \mathrm{mg} / \mathrm{dL}$ for females. A software program for guiding dosage adjustment to minimize the risk of drug accumulation was reported; ${ }^{25}$ however, it was not available at all locations. Therefore, the use of metformin in patients with mild renal impairment was subject to debate. Acarbose is a kind of $\alpha$-glucosidase inhibitor that delays the absorption of intestinal carbohydrates to achieve decreasing blood glucose. The relationship between the use of this medicine in CKD patients and the risk of ADEs was seldom reported. However, the MI clearly pointed out that acarbose should be avoided in patients with $\mathrm{Ccr}<25 \mathrm{~mL} / \mathrm{min}$. It is possible that drug accumulation may result in serious flatulence and diarrhea.
A Spanish university hospital reported that $54.1 \%$ of acute renal failure (ARF) cases were drug related. In addition, patients with drug-related ARF episodes were more prone to having cardiovascular events. The medicines most frequently involved were diuretics, agents acting on the renin-angiotensin system, immunosuppressant, $\beta$-blocking agents, calcium channel blockers, contrast media, and nonsteroidal anti-inflammatory drugs. ${ }^{32}$ Each of these medicines may affect renal function due to several mechanisms and, when used in combination, can lead to increased risk of ARF. So, these medicines should be used carefully in CKD patients. In this study, diuretic $(n=28)$, nonsteroidal anti-inflammatory drugs $(n=12)$, and angiotensin-converting enzyme inhibitors $(n=6)$ accounted for $1.62 \%, 0.69 \%$, and $0.35 \%$, respectively, of all prescriptions about inappropriate medication prescriptions. However, the combination of these medicines was not found in this study. It is worth reminding that these medicines should be carefully prescribed in CKD patients. Moreover, close monitoring should be emphasized.

It is worth reminding that nutraceutical and electrolytes were the most cautiously and contraindicated used medicines, including enteral nutritional emulsion, carbohydrateelectrolyte solution, potassium chloride, and so on. In CKD patients, drugs prone to accumulation may lead to electrolyte disturbance, such as hyperkalemia and hypercalcemia. Therefore, monitoring serum electrolyte levels should get particular attention. If the laboratory value exhibit in the normal level, this was not considered a potential risk factor.

\section{Limitations}

This study has a number of limitations. Even though it is a single-center study, the authors think the results may be extrapolated to other hospitals of similar characteristics. In addition, it is possible that some concealed CKD patients were omitted, because the inclusion criteria were based on serum creatinine and not on Ccr. Also, the study did not assess confounding factors such as patients' complication to medicine therapy. Finally, because it was a retrospective study, the relationship between inappropriate medicine use and occurrence of ADEs was not analyzed.

\section{Conclusion}

This study confirms that unreasonable medicine dosage, contraindicated, and cautiously used medicines were highly prevalent inappropriate prescriptions in CKD patients. With regard to these results, multifaceted measures should be applied, such as the integration of a decision support system about automatic hints and warnings into a computerized 
physician order entry system. Moreover, as reported, the intervention of a clinical pharmacist can also reduce the rates of inappropriate prescriptions. Putting into practice all the aforementioned facts could lead to an improvement in the quality of care provided to CKD patients.

\section{Acknowledgment}

The authors wish to thank all the participants in the study. This work was sponsored by National Science Foundation of China under contract 81671889.

\section{Disclosure}

The authors declare no potential conflicts of interest with respect to the research, authorship, and/or publication of this article.

\section{References}

1. Kirsztajn GM, Filho NS, Draibe SA, et al. Fast reading of the KDIGO 2012: guidelines for evaluation and management of chronic kidney disease in clinical practice. J Bras Nefrol. 2014;36(1):63-73.

2. Stevens PE, Levin A. Evaluation and management of chronic kidney disease: synopsis of the kidney disease: improving global outcomes 2012 clinical practice guideline. Ann Intern Med. 2013;158(11):825-830.

3. Collins AJ, Foley RN, Chavers B, et al. US Renal Data System 2013 Annual Data Report. Am J Kidney Dis. 2014;63(1 Suppl):A7.

4. Ojo A. Addressing the global burden of chronic kidney disease through clinical and translational research. Trans Am Clin Climatol Assoc. 2014; 125:229-243.

5. Manley HJ, Cannella CA, Bailie GR, St Peter WL. Medication-related problems in ambulatory hemodialysis patients: a pooled analysis. Am J Kidney Dis. 2005;46(4):669-680.

6. Farag A, Garg AX, Li L, Jain AK. Dosing errors in prescribed antibiotics for older persons with CKD: a retrospective time series analysis. $\mathrm{Am} J$ Kidney Dis. 2014;63(3):422-428.

7. Fink JC, Brown J, Hsu VD, Seliger SL, Walker L, Zhan M. CKD as an underrecognized threat to patient safety. Am J Kidney Dis. 2009;53(4): 681-688.

8. Fink JC, Chertow GM. Medication errors in chronic kidney disease: one piece in the patient safety puzzle. Kidney Int. 2009;76(11): $1123-1125$.

9. Ahmed B, Nanji K, Mujeeb R, Patel MJ. Effects of polypharmacy on adverse drug reactions among geriatric outpatients at a tertiary care hospital in Karachi: a prospective cohort study. PLoS One. 2014;9(11): e112133.

10. Franz CC, Egger S, Born C, Rätz Bravo AE, Krähenbühl S. Potential drug-drug interactions and adverse drug reactions in patients with liver cirrhosis. Eur J Clin Pharmacol. 2012;68(2):179-188.

11. Joshua L, Devi PD, Guido S. Adverse drug reactions in nephrology ward inpatients of a tertiary care hospital. Indian J Med Sci. 2007;61(10): $562-569$

12. Stafford AC, Alswayan MS, Tenni PC. Inappropriate prescribing in older residents of Australian care homes. J Clin Pharm Ther. 2011; 36(1):33-44.

13. Hefner G, Stieffenhofer V, Gabriel S, et al. Side effects related to potentially inappropriate medications in elderly psychiatric patients under everyday pharmacotherapy. Eur J Clin Pharmacol. 2015;71(2): $165-172$.

14. Jones SA, Bhandari S. Prevalence of potentially inappropriate medication prescribing in elderly patients with chronic kidney disease. Postgrad Med J. 2013;89(1051):247-250.
15. MannE, Haastert B, Böhmdorfer B, Frühwald T IB, Roller-Wirnsberger R, Meyer G. Prevalence and associations of potentially inappropriate prescriptions in Austrian nursing home residents: secondary analysis of a cross-sectional study. Wien Klin Wochenschr. 2013;125(7-8): 180-188.

16. Fahimi F, Emami S, Rashid Farokhi F. The rate of antibiotic dosage adjustment in renal dysfunction. Iran J Pharm Res. 2012;11(1): $157-161$.

17. Arlicot N, Pourrat X, Bourgoin-Hérard H, Grassin J, Antier D. Are antibiotic drugs well prescribed in case of renal insufficiency? A retrospective study. Ren Fail. 2007;29(8):1055-1058.

18. Jiang SP, Xu YY, Ping-Yang, et al. Improving antimicrobial dosing in critically ill patients receiving continuous venovenous hemofiltration and the effect of pharmacist dosing adjustment. Eur J Intern Med. 2014;25(10):930-935.

19. Joosten H, Drion I, Boogerd KJ, et al. Optimising drug prescribing and dispensing in subjects at risk for drug errors due to renal impairment: improving drug safety in primary healthcare by low eGFR alerts. $B M J$ Open. 2013;3(1):e002068.

20. Deng L-X, Han F-X, Yun X, Wang M. [Investigation about antibiotic appropriateness use in patients with renal insufficiency]. Herald of Medicine. 2015;34(5):676-680. Chinese.

21. Li P, Li F-j, Liu Y-g. [Rationality in antibiotic use in patients with chronic renal insufficiency]. Eval Anal Drug-Use Hosp China. 2012;12(5): 425-427. Chinese.

22. Bilge U, Sahin G, Unluoglu I, Ipek M, Durdu M, Keskin A. Inappropriate use of nonsteroidal anti-inflammatory drugs and other drugs in chronic kidney disease patients without renal replacement therapy. Ren Fail. 2013;35(6):906-910.

23. Dörks M, Herget-Rosenthal S, Schmiemann G, Hoffmann F. Use of nonsteroidal anti-inflammatory drugs and renal failure in nursing home residents-results of the study "Inappropriate Medication in Patients with Renal Insufficiency in Nursing Homes". Wien Klin Wochenschr. 2016; 128(7-8):287-290.

24. Bhardwaja B, Carroll NM, Raebel MA, et al. Improving prescribing safety in patients with renal insufficiency in the ambulatory setting: the Drug Renal Alert Pharmacy (DRAP) program. Pharmacotherapy. 2011; 31(4):346-356.

25. Erler A, Beyer M, Petersen JJ, et al. How to improve drug dosing for patients with renal impairment in primary care - a cluster-randomized controlled trial. BMC Fam Pract. 2012;13:91.

26. Hassan Y, Al-Ramahi RJ, Aziz NA, Ghazali R. Impact of a renal drug dosing service on dose adjustment in hospitalized patients with chronic kidney disease. Ann Pharmacother. 2009;43(10):1598-1605.

27. Akbari A, Swedko PJ, Clark HD, et al. Detection of chronic kidney disease with laboratory reporting of estimated glomerular filtration rate and an educational program. Arch Intern Med. 2004;164(16):1788-1792.

28. Stevens LA, Fares G, Fleming J, et al. Low rates of testing and diagnostic codes usage in a commercial clinical laboratory: evidence for lack of physician awareness of chronic kidney disease. J Am Soc Nephrol. 2005; 16(8):2439-2448.

29. van Dijk EA, Drabbe NR, Kruijtbosch M, De Smet PA. Drug dosage adjustments according to renal function at hospital discharge. Ann Pharmacother. 2006;40(7-8):1254-1260.

30. Sweileh WM, Janem SA, Sawalha AF, et al. Medication dosing errors in hospitalized patients with renal impairment: a study in Palestine. Pharmacoepidemiol Drug Saf. 2007;16(8):908-912.

31. Runge S, Mayerle J, Warnke C, et al. Metformin-associated lactic acidosis in patients with renal impairment solely due to drug accumulation? Diabetes Obes Metab. 2008;10(1):91-93.

32. Iavecchia L, Cereza García G, Sabaté Gallego M, et al. Drug-related acute renal failure in hospitalised patients. Nefrologia. 2015;35(6): $523-532$. 


\section{Publish your work in this journal}

Therapeutics and Clinical Risk Management is an international, peerreviewed journal of clinical therapeutics and risk management, focusing on concise rapid reporting of clinical studies in all therapeutic areas, outcomes, safety, and programs for the effective, safe, and sustained use of medicines. This journal is indexed on PubMed Central, CAS,

EMBase, Scopus and the Elsevier Bibliographic databases. The manuscript management system is completely online and includes a very quick and fair peer-review system, which is all easy to use. Visit $\mathrm{http}: / / \mathrm{www}$.dovepress.com/testimonials.php to read real quotes from published authors.

Submit your manuscript here: http://www.dovepress.com/therapeutics-and-clinical-risk-management-journal 